\title{
Existence and Nonexistence of Global Solutions of a Fully Nonlinear Parabolic Equation
}

\author{
Zhihao Ge \\ Institute of Applied Mathematics, School of Mathematics and Information Sciences, \\ Henan University, Kaifeng, China \\ Email: zhihaoge@henu.edu.cn
}

Received August 3, 2012; revised October 2, 2012; accepted October 12, 2012

\begin{abstract}
In the paper, we study the global existence of weak solution of the fully nonlinear parabolic problem (1.1)-(1.3) with nonlinear boundary conditions for the situation without strong absorption terms. Also, we consider the blow up of global solution of the problem (1.1)-(1.3) by using the convexity method.
\end{abstract}

Keywords: Nonlinear Parabolic Equation; Blow Up; Convexity Method

\section{Introduction}

In this paper, we consider the following fully nonlinear parabolic problem:

$$
\begin{gathered}
u_{t}=\Delta \varphi(u(x, t))-\lambda f(u(x, t)),(x, t) \in \Omega \times(0, T], \\
\frac{\partial \varphi(u(x, t))}{\partial v}=g(u(x, t)),(x, t) \in \partial \Omega \times(0, T], \\
u(x, 0)=u_{0}(x), x \in \bar{\Omega},
\end{gathered}
$$

where $\Omega$ is a bounded open domain with smooth boundary $\partial \Omega, \partial / \partial v$ is differentiation in the direction of the outward unit normal to $\partial \Omega, \lambda>0$ and $u_{0}(x) \in L^{\infty}(\Omega)$.

Denote $\varphi(u(x, t)), f(u(x, t))$ and $g(u(x, t))$ by $\varphi(u), \quad f(u)$, respectively. Also, we need the following conditions:

(D1) $f(s)$ and $g(s)$ are local Lipschiz continuous with respect to $s$;

(D2) $f(s)$ and $g(s)$ are positive for all $s$;

(D3) $\varphi(s) \in C^{1}(\mathbb{R})$ and $\varphi^{\prime}(s)>0$ with $\varphi(0)=0$.

The problem (1.1)-(1.3) appears in mathematical models of a number of areas of science such as gas dynamics, fluid flow, porous media and biological populations, one can see [1-9]. As for the case of semi-linear or degenerate equations with a nonlinear boundary condition which can be taken as the special case of the problem (1.1)(1.3), the behavior properties of the above mentioned such as existence and uniqueness, blow up of some special problems, have been established by [2,10-17] and so on.

In this paper, we study the conditions for global exis- tence and blow up of the problem (1.1)-(1.3). The remaining parts of the paper are organized as follows. In Section 2, we give the global solvability condition for the situations with and without strong absorption terms. Finally, we obtain the condition of blowing up of global solution by the convexity method in $[18,19]$.

\section{Global Existence}

Firstly, we give the definition of weak solution as follows:

Definition 2.1. Given $u_{0}(x) \in L^{\infty}(\Omega)$, if

$$
u(x, t) \in C\left([0, T] ; L^{1}(\Omega)\right) \cap L^{\infty}(0, T)
$$

satisfies

$$
\begin{aligned}
& \iint_{\Omega \times(0, T)}\left(\nabla \varphi(u) \nabla \phi-u \phi_{t}+\lambda f(u) \phi\right) \mathrm{d} x \mathrm{~d} t \\
& -\iint_{\partial \Omega \times(0, T)} g(u) \phi \mathrm{d} S_{x} \mathrm{~d} t=\int_{\Omega} u_{0}(x) \phi(x, 0) \mathrm{d} x
\end{aligned}
$$

for any test function

$$
\phi \in L^{2}\left(0, T ; H^{1}(\Omega)\right) \cap W^{1,1}\left(0, T ; L^{1}(\Omega)\right)
$$

with $\phi(T)=0$, then $u(x, t)$ is called by a weak solution of the problem (1.1), (1.2).

The local existence and uniqueness of weak solution of the problem (1.1)-(1.3), one can see [20]. For the global existence of weak solution, we have the following result:

Theorem 2.1. Assume that there exist strictly non-decreasing positive functions $H(s)$ and $H^{*}(s)$ such that 


$$
\begin{aligned}
& \varphi^{\prime}(s) H(s) \geq g(s) \text { for } s \geq s_{0}>0, \\
& H^{*}(s) \geq \varphi^{\prime}(s) l-\left(\varphi^{\prime}(s) H^{\prime}(s)+\varphi^{\prime \prime}(s) H(s)\right) M_{2}^{2} \\
&+\lambda f(s) / H(s),
\end{aligned}
$$

where

$$
\begin{aligned}
& M_{1}=\max _{\bar{\Omega}} h(x), \\
& M_{2}=\max _{\bar{\Omega}}|\nabla h(x)|
\end{aligned}
$$

and $h(x)$ satisfies

$$
\Delta h(x)=l \text { in } \Omega, \frac{\partial h(x)}{\partial v}=1 \text { on } \partial \Omega .
$$

Then the solution of the problem (1.1)-(1.3) is global.

Proof. Let $\bar{u}(x, t)=\psi(\delta(t)+h(x))$, where $\psi$ is the solution of

$$
\begin{aligned}
& \psi^{\prime}(s)=H(\psi(s)) \text { with } \\
& \psi(0)=\psi_{0} \geq\left\|u_{0}\right\|_{L^{\infty}} \geq s_{0},
\end{aligned}
$$

and $\delta(t)$ satisfies

$$
\begin{aligned}
& \delta^{\prime}(t)=H^{*}\left(\psi\left(\delta(t)+M_{1}\right)\right) \text { with } \\
& \delta(0)=0 .
\end{aligned}
$$

From (2.2), (2.3) and (2.6), (2.7), it follows that $\psi(\delta(t)+h(x))$ and $\delta(t)$ are well posed, positive and increasing for all $t \geq 0$.

Thus, there holds

$$
\begin{aligned}
& \bar{u}(x, t) \in C([0, \infty) \times \bar{\Omega}) \text { and } \\
& \bar{u}(x, t) \geq \psi_{0} \geq s_{0} .
\end{aligned}
$$

Using (2.5)-(2.7) and (2.3), we have

$$
\begin{aligned}
\bar{u}_{t}-\Delta \varphi(\bar{u})+\lambda f(\bar{u}) & =H(\bar{u}) \delta^{\prime}(t)-\Delta \varphi(\bar{u})+\lambda f(\bar{u}) \\
& =H(\bar{u}) \delta^{\prime}(t)-\left[\varphi^{\prime}(\bar{u}) H(\bar{u}) l+\left(\varphi^{\prime}(\bar{u}) \psi^{\prime \prime}(\delta(t)+h(x))+\varphi^{\prime \prime}(\bar{u}) H^{2}(\bar{u})\right)\left|\nabla h^{2}\right|\right]+\lambda f(\bar{u}) \\
& =H(\bar{u}) \delta^{\prime}(t)-\left[\varphi^{\prime}(\bar{u}) H(\bar{u}) l+\left(\varphi^{\prime}(\bar{u}) H(\bar{u}) H^{\prime}(\bar{u})\right)+\varphi^{\prime \prime}(\bar{u}) H^{2}(\bar{u})\left|\nabla h^{2}\right|\right]+\lambda f(\bar{u}) \\
& \geq H(\bar{u})\left[\delta^{\prime}(t)-\varphi^{\prime}(\bar{u}) l-\left(\varphi^{\prime}(\bar{u}) H^{\prime}(\bar{u})+\varphi^{\prime \prime}(\bar{u}) H(\bar{u}) M_{2}^{2}+\lambda f(\bar{u}) / H(\bar{u})\right)\right] \\
& \geq H(\bar{u})\left[H^{*}(\bar{u})-\varphi^{\prime}(\bar{u}) l-\left(\varphi^{\prime}(\bar{u}) H^{\prime}(\bar{u})+\varphi^{\prime \prime}(\bar{u}) H(\bar{u}) M_{2}^{2}+\lambda f(\bar{u}) / H(\bar{u})\right)\right] \geq 0 .
\end{aligned}
$$

Using (2.2), (2.5) and (2.6), we obtain

$$
\begin{aligned}
\frac{\partial \varphi(\bar{u})}{\partial v}-g(\bar{u}) & =\nabla \varphi(\bar{u}) \cdot \mathbf{n}_{0}-g(\bar{u})=\varphi^{\prime}(\bar{u}) \nabla \psi(\delta(t)+h(x)) \cdot \mathbf{n}_{0}-g(\bar{u}) \\
& =\varphi^{\prime}(\bar{u}) H(\bar{u}) \nabla h \cdot \mathbf{n}_{0}-g(\bar{u})=\varphi^{\prime}(\bar{u}) H(\bar{u}) \partial h / \partial v-g(\bar{u})=\varphi^{\prime}(\bar{u}) H(\bar{u})-g(\bar{u}) \geq 0 .
\end{aligned}
$$

From (2.9) and (2.10), we see that $\bar{u}(x, t)$ is a supsolution to the problem (1.1)-(1.3) defined for all $t \geq 0$ with $\bar{u}(x, 0) \geq u_{0}(x)$. By using the sup- and sub-solution argument (c.f. [7]), we know that the solution o the problem (1.1)-(1.3) is global.

Remark 2.1. If the conditions (2.2) and (2.3) hold, the problem (1.1)-(1.3) is called by the problem without strong absorption terms.

\section{Blow Up}

In the section, we use the convexity method (see $[18,19]$ ) to show that the global solution blows up in finite time under some suitable condition. To this end, we define

$$
E(t)=-\frac{1}{2} \int_{\Omega}|\nabla \varphi(u)|^{2} \mathrm{~d} x+\frac{1}{2} \int_{\partial \Omega} \int_{0}^{u} \varphi^{\prime}(s) \mathrm{d} s g(u) \mathrm{d} S_{x}-\lambda \int_{\Omega} \int_{0}^{u} \varphi^{\prime}(s) f(s) \mathrm{d} s \mathrm{~d} x
$$

and

$$
F(t)=\int_{\Omega} \int_{0}^{u} \varphi(z) \mathrm{d} z \mathrm{~d} x
$$

Suppose that following conditions hold:

(D4) If $g, \varphi$ and $f$ satisfy the following inequalities

$$
g(s) \int_{0}^{s} \varphi^{\prime}(z) \mathrm{d} z \geq 2 \int_{0}^{s} \varphi^{\prime}(z) g(z) \mathrm{d} z
$$

and

$$
2 f(s) \int_{0}^{s} \varphi^{\prime}(z) \mathrm{d} z \geq 2 \int_{0}^{s} \varphi^{\prime}(z) f(z) \mathrm{d} z .
$$

(D5) There exist a constant $I_{0}$ and a convexity function $\psi(s)>0$ such that

$$
\int_{I_{0} / / \Omega \mid}^{+\infty} \frac{\mathrm{d} s}{\psi(s)}<+\infty
$$

and

$$
\frac{2 E(0)}{|\Omega|}+\frac{\lambda}{2} \int_{0}^{s} \varphi^{\prime}(z) f(z) \mathrm{d} z \geq \psi\left(\int_{0}^{s} \varphi(z) \mathrm{d} z\right)>0
$$

with 


$$
\begin{aligned}
E(0)= & -\frac{1}{2} \int_{\Omega}\left|\nabla \varphi\left(u_{0}\right)\right|^{2} \mathrm{~d} x+\frac{1}{2} \int_{\partial \Omega} \int_{0}^{u_{0}} \varphi^{\prime}(s) \mathrm{d} s g\left(u_{0}\right) \mathrm{d} S_{x} \\
& -\lambda \int_{\Omega} \int_{0}^{u_{0}} \varphi^{\prime}(s) f(s) \mathrm{d} s \mathrm{~d} x .
\end{aligned}
$$

Lemma 3.1. If the condition (D4) holds, then $E(t) \geq E(0)$, i.e.,

$$
\begin{aligned}
& \frac{1}{2} \int_{\partial \Omega} \int_{0}^{u} \varphi^{\prime}(s) \mathrm{d} s g(u) \mathrm{d} x \\
& \geq E(0)+\frac{1}{2} \int_{\Omega}|\nabla \varphi(u)|^{2} \mathrm{~d} x+\lambda \int_{\Omega}^{u} \int_{0}^{u} \varphi^{\prime}(s) f(s) \mathrm{d} s \mathrm{~d} x .
\end{aligned}
$$

Proof. Multiplying (1.1) by $\varphi(u)_{t}$ and integrating by parts over $\Omega$, we have

$$
\begin{aligned}
0 & \leq \int_{\Omega} \varphi^{\prime}(u)\left(u_{t}\right)^{2} \mathrm{~d} x=\int_{\Omega} \varphi(u)_{t} \Delta \varphi(u) \mathrm{d} x-\lambda \int_{\Omega} f(u) \varphi(u)_{t} \mathrm{~d} x \\
& =-\int_{\Omega} \nabla \varphi(u) \nabla \varphi(u)_{t} \mathrm{~d} x+\int_{\partial \Omega} \varphi(u)_{t} g(u) \mathrm{d} S_{x}-\lambda \int_{\Omega} f(u) \varphi(u)_{t} \mathrm{~d} x \\
& =-\int_{\Omega} \nabla \varphi(u) \nabla \varphi(u)_{t} \mathrm{~d} x+\int_{\partial \Omega} \varphi^{\prime}(u) g(u) u_{t} \mathrm{~d} S_{x}-\lambda \int_{\Omega} f(u) \varphi^{\prime}(u) u_{t} \mathrm{~d} x \\
& =\frac{\mathrm{d}}{\mathrm{d} t}\left[-\int_{\Omega}\left(\frac{1}{2}|\nabla \varphi(u)|^{2}+\lambda \int_{0}^{u} \varphi^{\prime}(s) f(s) \mathrm{d} s\right) \mathrm{d} x+\int_{\partial \Omega}\left(\int_{0}^{u} \varphi^{\prime}(s) f(s) \mathrm{d} s\right) \mathrm{d} S_{x}\right] .
\end{aligned}
$$

Using (3.8), we have

$$
\frac{\mathrm{d}}{\mathrm{d} t}\left[-\frac{1}{2} \int_{\Omega}|\nabla \varphi(u)|^{2} \mathrm{~d} x+\frac{1}{2} \int_{\partial \Omega}\left(\int_{0}^{u} \varphi^{\prime}(s) \mathrm{d} s\right) g(u) \mathrm{d} S_{x}-\lambda \int_{\Omega}\left(\int_{0}^{u} \varphi^{\prime}(s) f(s) \mathrm{d} s\right) \mathrm{d} x\right] \geq 0 .
$$

Using (3.9) and (3.1), we have $\mathrm{d} E(t) / \mathrm{d} t \geq 0$. So, we obtain $E(t) \geq E(0)$.

Theorem 3.1. Suppose that the conditions (D4) and
(D5) hold, then the solution of the problem (1.1)-(1.3) blows up in finite time.

Proof. Using (3.2), we have

$$
\begin{aligned}
\frac{\mathrm{d} F(t)}{\mathrm{d} t} & =\int_{\Omega}\left(\int_{0}^{u} \varphi^{\prime}(z) \mathrm{d} z\right) u_{t} \mathrm{~d} x=\int_{\Omega}\left(\int_{0}^{u} \varphi^{\prime}(z) \mathrm{d} z\right) \Delta \varphi(u) \mathrm{d} x-\lambda \int_{\Omega}\left(\int_{0}^{u} \varphi^{\prime}(z) \mathrm{d} z\right) f(u) \mathrm{d} x \\
& =\int_{\partial \Omega}\left(\int_{0}^{u} \varphi^{\prime}(z) \mathrm{d} z\right) g(u) \mathrm{d} S_{x}-\int_{\Omega} \nabla\left(\int_{0}^{u} \varphi^{\prime}(z) \mathrm{d} z\right) \nabla \varphi(u) \mathrm{d} x-\lambda \int_{\Omega}\left(\int_{0}^{u} \varphi^{\prime}(z) \mathrm{d} z\right) f(u) \mathrm{d} x .
\end{aligned}
$$

Since $\varphi(u)=\int_{0}^{u} \varphi^{\prime}(s) \mathrm{d} s$, so we have

$$
\nabla \varphi(u)=\varphi^{\prime}(u) \nabla u \text {. }
$$

Multiplying (3.11) by $\nabla \varphi(u)$ and integrating over

\section{$\Omega$, we have}

$$
\int_{\Omega}|\nabla \varphi(u)|^{2} \mathrm{~d} x=\int_{\Omega} \varphi^{\prime}(u) \nabla u \nabla \varphi^{\prime}(u) \mathrm{d} x .
$$

Using (3.12) and Lemma 3.1, we obtain

$$
\begin{aligned}
F^{\prime}(t) & \geq 2 E(0)+\int_{\Omega}|\nabla \varphi(u)|^{2} \mathrm{~d} x+\lambda \int_{\Omega} \int_{0}^{u} \varphi^{\prime}(s) f(s) \mathrm{d} s \mathrm{~d} x-\int_{\Omega} \varphi^{\prime}(u) \nabla \varphi(u) \nabla u \mathrm{~d} x-\lambda \int_{\Omega} \int_{0}^{u} \varphi^{\prime}(z) \mathrm{d} z f(u) \mathrm{d} x \\
& =2 E(0)+\lambda \int_{\Omega} \int_{0}^{u} \varphi^{\prime}(s) f(s) \mathrm{d} s \mathrm{~d} x-\lambda \int_{\Omega} \int_{0}^{u} \varphi^{\prime}(z) \mathrm{d} z f(u) \mathrm{d} x=2 E(0)+\frac{\lambda}{2} \int_{\Omega} \int_{0}^{u} \varphi^{\prime}(s) f(s) \mathrm{d} s \mathrm{~d} x \\
& =\int_{\Omega}\left(\frac{\lambda}{2} \int_{0}^{u} \varphi^{\prime}(s) f(s) \mathrm{d} s+\frac{2 E(0)}{|\Omega|}\right) \mathrm{d} x .
\end{aligned}
$$

From the condition (D5), we see

$$
F^{\prime}(t) \geq \int_{\Omega} \psi\left(\int_{0}^{u} \varphi(z) \mathrm{d} z\right) \mathrm{d} x>0 .
$$

Using the Jensen's inequality, we get

$$
\begin{aligned}
F^{\prime}(t) & \geq|\Omega| \psi\left(\int_{\Omega} \int_{0}^{u} \varphi(z) \mathrm{d} z \mathrm{~d} x /|\Omega|\right) \\
& =|\Omega| \psi(F(t) /|\Omega|) .
\end{aligned}
$$

Hence, we have

$$
F^{\prime}(t) /|\Omega| \psi(F(t) /|\Omega|) \geq 1 .
$$

Integrating (3.16) from 0 to $t$, we have

$$
\int_{0}^{t} F^{\prime}(\tau) \mathrm{d} \tau /(|\Omega| \psi(F(\tau) /|\Omega|)) \geq t .
$$

Let $y=F(t) /|\Omega|$, then (3.17) becomes

$$
\int_{F(0) / \Omega \mid}^{F(t) / \Omega \mid} \frac{\mathrm{d} y}{\psi(y)} \geq t .
$$

By the condition (D5), we have 


$$
\int_{F(0) / \Omega \Omega \mid}^{+\infty} \frac{\mathrm{d} y}{\psi(y)}<+\infty
$$

Therefore, there exists $T_{0}$ such that

$$
\lim _{t \rightarrow T_{0}^{-}} F(t)=\lim _{t \rightarrow T_{0}^{-}} \int_{\Omega}\left(\int_{0}^{u} \psi(z) \mathrm{d} z\right) \mathrm{d} x=+\infty .
$$

From (3.20), we know that the solution of the problem (1.1)-(1.3) must blow up in finite time.

\section{Acknowledgements}

The present work is supported by National Natural Science Foundation of China under Grant No. 10901047.

\section{REFERENCES}

[1] H. Amann, "Periodic Solutions of Semi-Linear Parabolic Equations,” Academic Press, New York, San Francisco, London, 1978.

[2] A. Friedman and B. Mcleod, "Blow Up of Solutions of Semilinear Heat Equations,” Indiana University Mathematics Journal, Vol. 34, No. 1, 1985, pp. 425-447. doi:10.1512/iumj.1985.34.34025

[3] V. Galaktionov and J. Vazquez, "The Problem of Blow Up in Nonlinear Parabolic Equations,” Discrete and Continuous Dynamical Systems, Vol. 8, No. 2, 2002, pp. 399433. doi:10.3934/dcds.2002.8.399

[4] X. Song and S. Zheng, "Multinonlinear Interactions in Quasi-Linear Reaction-Diffusion Equations with Nonlinear Boundary Flux,” Mathematical and Computer Modelling, Vol. 39, No. 2-3, 2004, pp. 133-144. doi:10.1016/S0895-7177(04)90002-7

[5] D. G. Aronson, “The Porous Medium Equation,” Springer Verlag, Berlin, 1986.

[6] M. Gurtin and R. Cammy, "On the Diffusion of Biological Population,” Mathematical and Theoretical Biology, Vol. 33, No. 1-2, 1977, pp. 35-49.

[7] C. V. Pao, "Nonlinear Parabolic and Elliptic Equations," Plenum Press, New York, 1992.

[8] O. Ladyzenskaja, V. Solonnikov and N. Uralceva, "Linear and Quasilinear Equations of Parabolic Type,” American Mathematical Society, Providence, 1968.

[9] R. Temam, "Infinite Dimensional Systems in Mechanics and Physics,” Springer, New York, 1997.
[10] P. Quittner and P. Souplet, "Bounds of Global Solutions of Parabolic Problems with Nonlinear Boundary Conditions," Indiana University Mathematics Journal, Vol. 52, No. 4, 2003, pp. 875-900. doi:10.1512/iumj.2003.52.2353

[11] P. Quittner and P. Souplet, “A Priori Estimates of Global Solutions of Superlinear Parabolic Problems without Variational Structure,” Discrete and Continuous Dynamical Systems, Vol. 9, No. 5, 2003, pp. 1277-1292. doi:10.3934/dcds.2003.9.1277

[12] A. Bernal and A. Tajdine, "Nonlinear Balance for Reaction-Diffusion Equations under Nonliear Boundary Conditions: Dissipativity and Blow Up,” Journal of Differential Equation, Vol. 169, No. 2, 2001, pp. 332-372. doi:10.1006/jdeq.2000.3903

[13] J. D. Rossi, "The Blow-Up Rate for a Semilinear Parabolic Equation with a Nonlinear Boundary Condition," Acta Mathematica Universitatis Comenianae, Vol. 67, No. 2, 1998, pp. 343-350.

[14] J. D. Rossi and N. Wolanski, "Global Existence and Nonexistence for a Parabolic System with Nonlinear Boundary Conditions,” Differential and Integral Equations, Vol. 11, No. 1, 1998, pp. 179-190.

[15] A. Samarskii, V. Galaktionov and S. Mikhailov, "BlowUp in Quasilinear Parabolic Equations,” Walter de Gruyter, Berlin/New York, 1995. doi:10.1515/9783110889864

[16] K. Taira, "Semi-Linear Parabolic Problems in Combustion Theory," Journal of Mathematical Sciences-The University of Tokyo, Vol. 10, No. 3, 2003, pp. 455-494.

[17] J. Filo and P. D. Mottoni, “Global Existence and Decay of Solutions of the Porous Medium Equation with Nonlinear Boundary Conditions," Communications in Partial Differential Equations, Vol. 17, No. 5-6, 1992, pp. 737-765. doi:10.1080/03605309208820862

[18] H. A. Levine, "Instability and Nonexistence of Global Solutions of Nonlinear Wave Equations of the Form $P u_{t t}=A u+F(u)$," Transactions of the American Mathematical Society, Vol. 192, 1974, pp. 1-21.

[19] H. A. Levine, "Some Additional Remarks on the Nonexistence of Global Solutions to Nonlinear Wave Equations," SIAM Journal on Mathematical Analysis, Vol. 5, No. 1, 1974, pp. 138-146. doi:10.1137/0505015

[20] Z. H. Ge and Y. N. He, "Global Existence of Solutions of a Nonlinear Degenerate Parabolic Problem," Journal of Physics: Conference Series, Vol. 96, No. 1, 2008, Article ID: 012024. 